\title{
Effect of Microwave Irradiation on Oximation of Acetylferrocene
}

\author{
Yutaka Okada*, Ryuichi Maeda \\ Department of Applied Chemistry, Ritsumeikan University, Shiga, Japan \\ Email: *ygvictor@sk.ritsumei.ac.jp
}

How to cite this paper: Okada, Y. and Maeda, R. (2021) Effect of Microwave Irradiation on Oximation of Acetylferrocene. Green and Sustainable Chemistry, 11, 1-8. https://doi.org/10.4236/gsc.2021.111001

Received: December 24, 2020

Accepted: January 29, 2021

Published: February 2, 2021

Copyright $\odot 2021$ by author(s) and Scientific Research Publishing Inc. This work is licensed under the Creative Commons Attribution International License (CC BY 4.0).

http://creativecommons.org/licenses/by/4.0/

\begin{abstract}
Microwave-assisted reactions are an environmentally friendly approach for synthesizing organic compounds. In this study, oximation of acetylferrocene and acetophenone was conducted under both microwave irradiation and conventional heating conditions. Acetylferrocene and acetophenone were subjected to oximation under the two conditions in various solvent mixtures, and the extent of conversion was determined by ${ }^{1} \mathrm{H}$ nuclear magnetic resonance spectroscopy. Microwave irradiation was found to accelerate the rate of oximation of both acetylferrocene and acetophenone. Acceleration of the reaction under microwave irradiation was attributed to the efficient absorption of microwaves by the ferrocene nucleus.
\end{abstract}

\section{Keywords}

Microwave Irradiation, Conventional Heating, Ferrocene Nucleus, Acetophenone, Acetylferrocene, Oximation

\section{Introduction}

Environmentally friendly synthesis is a major concern in synthetic organic chemistry today [1] [2] [3] [4]. To conduct an environmentally friendly synthesis, the use of clean reagents and clean solvents such as water or supercritical fluids is essential. It is also important to use clean sources to drive the reactions. These sources include light, electricity, and catalysts.

Organic reactions initiated by microwave irradiation proceed extremely fast, giving products in high yields and high selectivities [5]. The use of microwaves is an environmentally friendly approach as it does not generate by-products. Moreover, the reaction is completed in a short time, because of which this is an energy-saving approach too. Organic synthesis using microwave irradiation was first reported in 1986 [6]. The reaction time was significantly shorter than that 
required using conventional heat sources such as an oil bath. Unlike conventional heating, heating by microwaves generates heat from the interior of the molecule. Therefore, in a solvent with a large dielectric loss, the energy generated by microwaves becomes larger than the energy dissipated, and a phenomenon called superheating occurs, in which the solvent is heated above its boiling point. The formation of a local spot, also known as a hot spot, is considered to be one of the factors accelerating the reaction. Other factors such as an increased frequency factor and decreased activation energy, as suggested by the reaction rate equation, have been proposed.

In addition, selective absorption of microwaves by protic solvents, polar substrates, and polar reaction intermediates is considered to be a major factor contributing to the increased reaction rates under microwave irradiation [7].

Oximes are an industrially useful compound and are employed as a starting material for the Beckmann rearrangement [8], which involves the conversion to nitrile via a dehydration reaction [9]. Thus, it is indispensable to develop a method for conducting the oximation in a short time and obtain the products in high yields.

The purpose of this study was to synthesize acetylferrocene oxime under conventional heating conditions and microwave irradiation (Scheme 1) and compare their yields. The effect of microwave absorption by the solvent was also examined. We also investigated the effect of microwave irradiation on the ferrocene nucleus by performing the same reaction using acetophenone. The mechanisms of the microwave irradiation-assisted chemical reactions were investigated.

\section{Experimental}

\subsection{Substrates}

Acetophenone was purchased from Nacalai Tesque, LTD.

\section{Synthesis of Acetylferrocene [10]}

In a $1000 \mathrm{~mL}$ three-necked flask attached with a condenser, $37.2 \mathrm{~g}(0.20 \mathrm{~mol})$ of ferrocene, $108.6 \mathrm{~g}(1.06 \mathrm{~mol})$ of acetic anhydride, and $8.0 \mathrm{~mL}(1.6 \mathrm{~mol})$ of phosphoric acid were added. The mixture was placed in an oil bath at $100^{\circ} \mathrm{C}$ for 10 min and stirred for the reaction to proceed. Following this, the mixture was cooled with ice water and neutralized with $500 \mathrm{~mL}$ of a $1 \mathrm{~N}$ aqueous sodium carbonate solution. The precipitate formed was filtered under suction using a

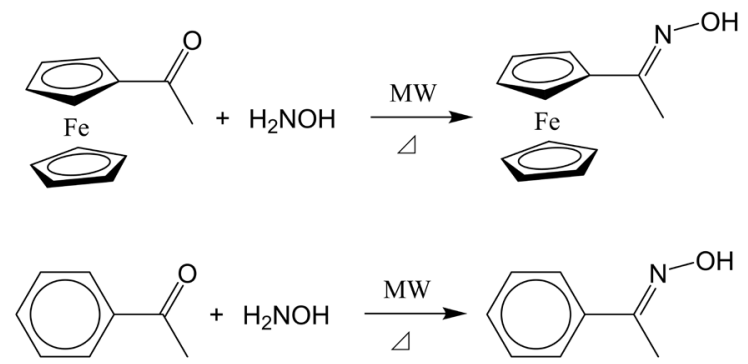

Scheme 1. Oximation of acetylferrocene and acetophenone. 
Nutche filter, washed with water until there was no odor of acetic acid, and dried overnight in a desiccator. The reaction was dissolved in a small amount of toluene, and impurities were removed by column chromatography using a column packed with $400 \mathrm{~g}$ of activated alumina (200 mesh). Toluene was used as the developing solution, and it was distilled off from the solution to obtain the product.

\subsection{Oximation}

\subsubsection{Conventional Heating Conditions}

Acetylferrocene $(0.7 \mathrm{mmol}, 0.16 \mathrm{~g})$ and hydroxylamine hydrochloride (0.7 $\mathrm{mmol}, 0.054 \mathrm{~g}$ ) were dissolved in $20 \mathrm{~mL}$ of various solvents. The solution was placed in a $50 \mathrm{~mL}$ three-necked flask and heated and stirred in an oil bath (1). The temperature was set to the maximum temperature possible under microwave irradiation. After the reaction, the mixture was cooled to room temperature, extracted with diethyl ether, and washed with water three times. Then, magnesium sulfate was added to the organic layer and dried. Following this, pleated filtration was performed to evaporate the filtrate. The desired product was isolated by column chromatography using hexane-methyl acetate mixed solvent. The same method was used for the oximation of acetophenone.

\subsubsection{Microwave Irradiation Conditions}

A $20 \mathrm{~mL}$ solution of $0.7 \mathrm{mmol}(0.16 \mathrm{~g})$ of acetylferrocene and $0.7 \mathrm{mmol}(0.054 \mathrm{~g})$ of hydroxylamine hydrochloride in various solvents was placed in a $50 \mathrm{~mL}$ three-necked flask. The solutions were subjected to microwave irradiation at 100 $\mathrm{W}$ for any time for the reaction to proceed. After completion of the reaction, the mixture was cooled to room temperature, extracted with diethyl ether, and washed with water three times. Then, magnesium sulfate was added to the organic layer and dried. Following this, pleated filtration was performed to evaporate the filtrate. The desired product was isolated by column chromatography using a hexane-ethyl acetate mixed solvent. The same method was used for the oximation of acetophenone.

\subsection{The Measuring Equipment}

The ${ }^{1} \mathrm{H}$ nuclear magnetic resonance was measured in chloroform-d at room temperature using a JEOL ECS-400 spectrometer.

\section{Results and Discussion}

To compare the results under microwave irradiation conditions and conventional heating conditions, the reactions were performed under the same conditions as much as possible. To achieve the same reaction temperature, all reactions were carried out under reflux conditions.

\subsection{Extent of Conversion under Conventional Heating Conditions and Microwave Irradiation}

The reaction was conducted in a pyridine:ethanol (1:1) mixed solvent. Figure 1 
and Figure 2 show the yield of acetylferrocene oxime and acetophenone oxime, respectively. The yields for both acetylferrocene oxime and acetophenone oxime were higher under microwave irradiation, suggesting that microwaves could accelerate the reaction.

\subsection{Effect of Microwave Irradiation on Ferrocene Nucleus}

We investigated the effect of microwave irradiation on the ferrocene nucleus. Pyridine:1-octanol mixed solvents (1:1, 1:3, and 1:20) were used for the reaction. When a higher alcohol, i.e., octanol, was used in the solvent mixture, the low-polarity solvent did not absorb microwaves to a considerable extent [7]. Consequently, microwaves were selectively absorbed by the substrate. As a typical example, the yields in 1:3 pyridine-1-octanol mixture are shown in Figure 3 and Figure 4. The yield of oxime obtained from acetylferrocene was slightly higher under microwave irradiation. On the other hand, there was no significant difference in the yields obtained through conventional heating and microwave irradiation when acetophenone was used as the starting compound.

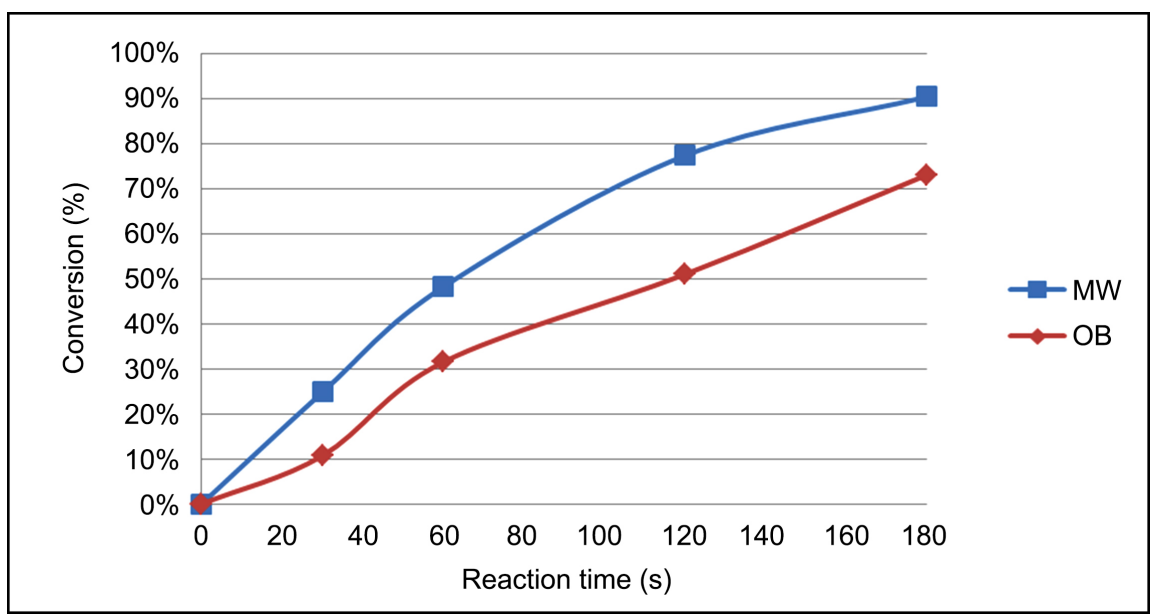

Figure 1. Yield of acetylferrocene oxime as a function of time (pyridine:ethanol =1:1).

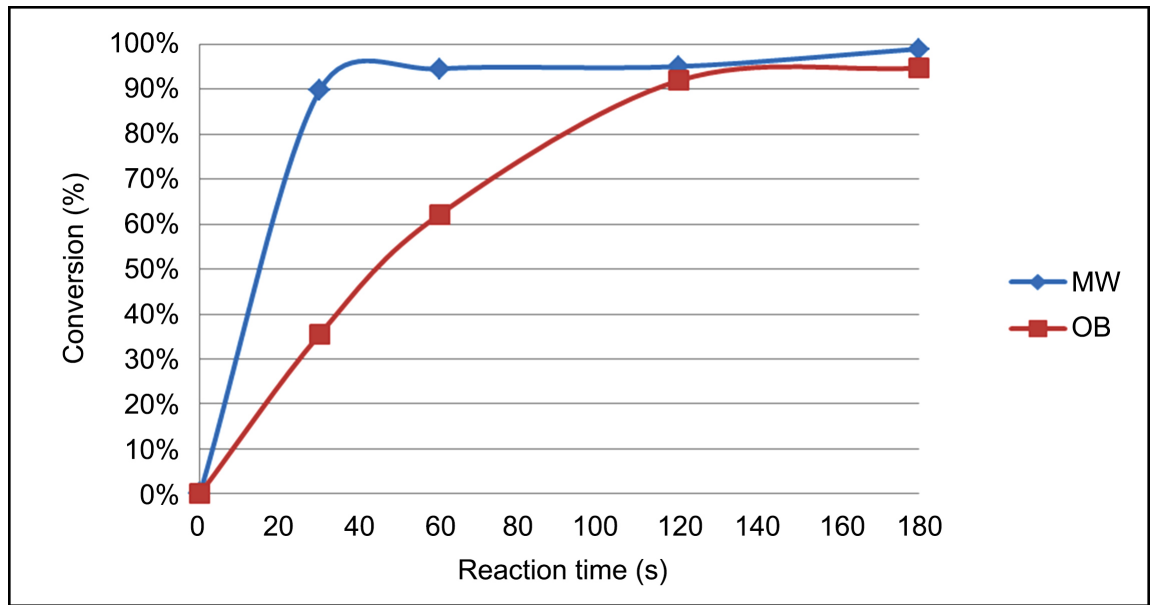

Figure 2. Yield of acetophenone oxime as a function of time (pyridine:ethanol = 1:1). 


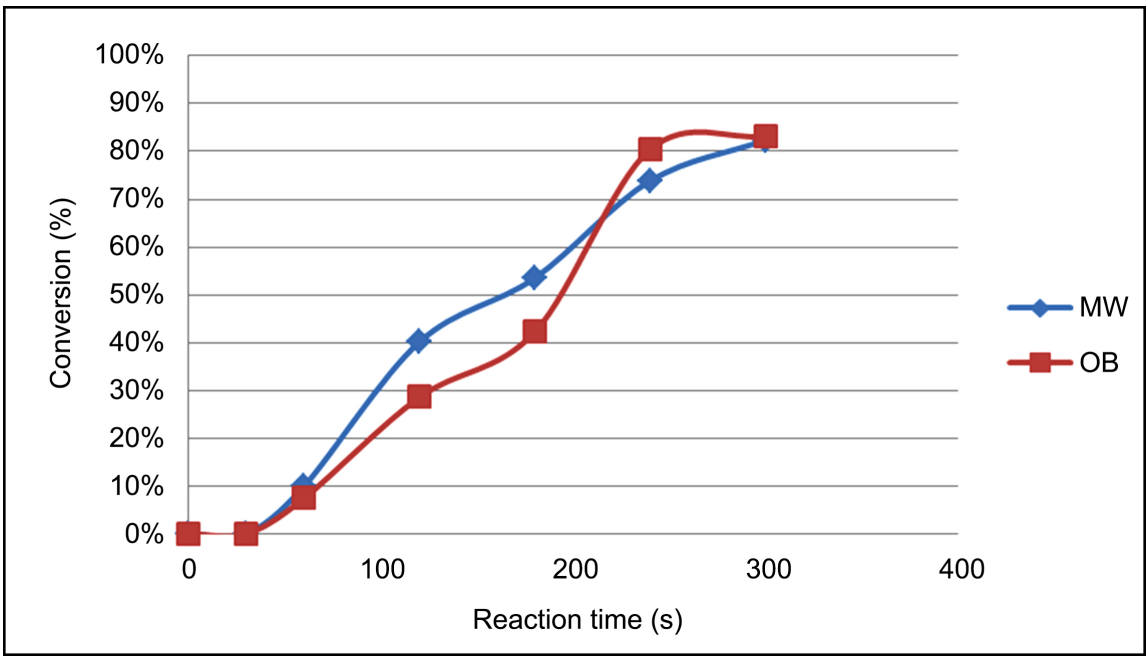

Figure 3. Yield of acetylferrocene oxime as a function of time (pyridine:1-octanol = 3:1).

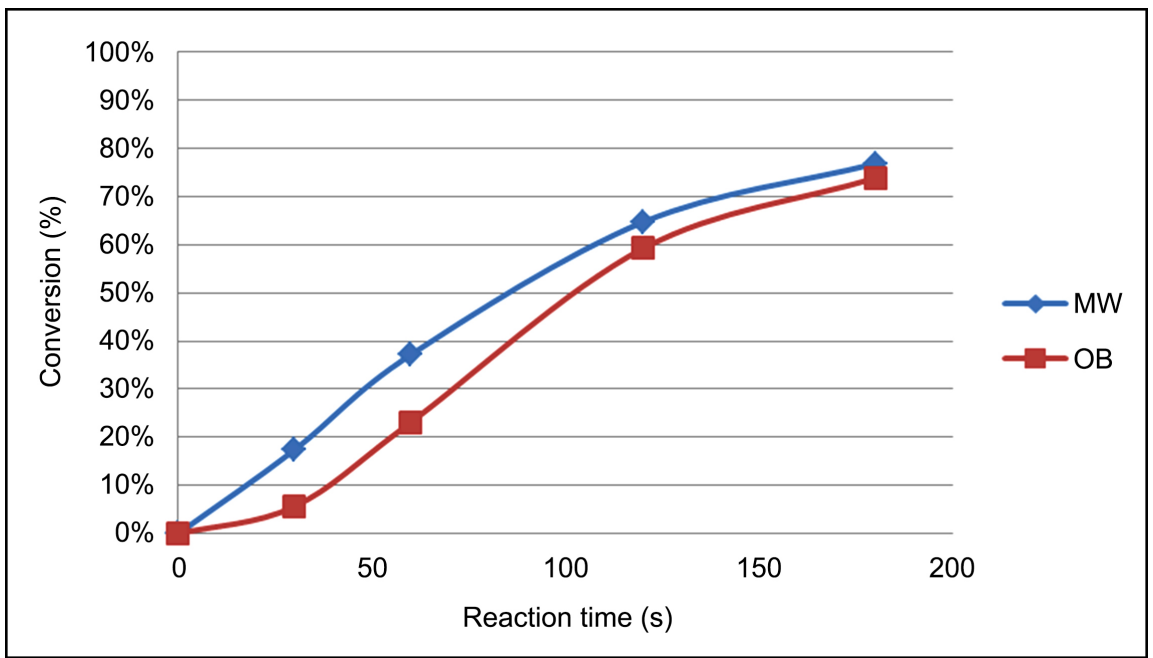

Figure 4. Yield of acetophenone oxime as a function of time (pyridine:1-octanol = 3:1).

Therefore, we investigated the effect of microwaves under mild conditions by reducing the amount of hydroxylamine hydrochloride. Table 1 and Table 2 show the results with acetylferrocene and acetophenone, respectively. Under microwave irradiation, the yield decreased by only a few percent when the amount of hydroxylamine hydrochloride was decreased (Entry 1 and 2, Table 1). However, under conventional heating conditions, the yields decreased significantly when the amount of hydroxylamine hydrochloride was decreased (Entry 6 and 5). Noticeably, the yield under conventional heating conditions was higher than that under microwave irradiation (Entry 2 and 6). However, when the amount of hydroxylamine hydrochloride was halved, the yield under microwave irradiation was slightly higher than that under conventional heating conditions, despite the unfavorable temperature (Entry 1 and 5). A remarkably higher yield was obtained even when the microwave output was increased to $300 \mathrm{~W}$ (Entry 2 and 4). 
Table 1. Yields from the oximation of acetylferrocene.

\begin{tabular}{|c|c|c|c|c|c|}
\hline Entry & Condition $^{\mathrm{a}}$ & $\begin{array}{c}\text { Reaction } \\
\text { time (s) }\end{array}$ & $\begin{array}{c}\text { Temperature } \\
\left({ }^{\circ} \mathrm{C}\right)\end{array}$ & $\begin{array}{l}\mathrm{H}_{2} \mathrm{NOH} \\
(\mathrm{mmol})\end{array}$ & $\begin{array}{c}\text { Conversion } \\
(\%)^{\mathrm{b}}\end{array}$ \\
\hline 1 & Irradiation of MW $(100 \mathrm{~W})$ & 240 & 68 & 0.35 & $\begin{array}{c}16 \\
\text { syn:anti }=0: 100\end{array}$ \\
\hline 2 & Irradiation of MW $(100 \mathrm{~W})$ & 240 & 68 & 0.70 & $\begin{array}{c}19 \\
\text { syn:anti }=11: 89\end{array}$ \\
\hline 3 & Irradiation of MW $(100 \mathrm{~W})$ & 240 & 68 & 1.40 & $\begin{array}{c}55 \\
\text { syn:anti }=13: 87\end{array}$ \\
\hline 4 & Irradiation of MW ( $300 \mathrm{~W})$ & 120 & 113 & 0.70 & $\begin{array}{c}92 \\
\text { syn:anti }=5: 95\end{array}$ \\
\hline 5 & Conventional heating & 240 & 68 & 0.35 & $\begin{array}{c}14 \\
\text { syn:anti }=0: 100\end{array}$ \\
\hline 6 & Conventional heating & 240 & 68 & 0.70 & $\begin{array}{c}34 \\
\text { syn:anti }=21: 79\end{array}$ \\
\hline 7 & Conventional heating & 240 & 68 & 1.40 & $\begin{array}{c}51 \\
\text { syn:ant }=8: 92\end{array}$ \\
\hline 8 & Conventional heating & 120 & 113 & 0.70 & $\begin{array}{c}91 \\
\text { syn:anti }=10: 90\end{array}$ \\
\hline
\end{tabular}

${ }^{a}$ Solvent is pyridine: 1 -octanol $=1: 20 ;{ }^{b}$ Conversion determined by $1 \mathrm{H}-\mathrm{NMR}$ integrated value.

Table 2. Yields from the oximation of acetophenone.

\begin{tabular}{|c|c|c|c|c|c|}
\hline Entry & Condition $^{\mathrm{a}}$ & $\begin{array}{l}\text { Reaction } \\
\text { time (s) }\end{array}$ & $\begin{array}{c}\text { Temperature } \\
\left({ }^{\circ} \mathrm{C}\right)\end{array}$ & $\begin{array}{l}\mathrm{H}_{2} \mathrm{NOH} \\
(\mathrm{mmol})\end{array}$ & $\begin{array}{c}\text { Conversion } \\
(\%)^{\mathrm{b}}\end{array}$ \\
\hline 1 & Irradiation of MW $(100 \mathrm{~W})$ & 240 & 68 & 0.35 & $\begin{array}{c}45 \\
\text { syn:ant } i=20: 80\end{array}$ \\
\hline 2 & Irradiation of MW (100 W) & 240 & 68 & 0.70 & $\begin{array}{c}65 \\
\text { syn:anti }=23: 77\end{array}$ \\
\hline 3 & Irradiation of MW (100 W) & 240 & 68 & 1.40 & $\begin{array}{c}97 \\
\text { syn:anti }=11: 89\end{array}$ \\
\hline 4 & Irradiation of MW ( $300 \mathrm{~W})$ & 120 & 113 & 0.70 & $\begin{array}{c}61 \\
\text { syn:anti }=20: 80\end{array}$ \\
\hline 5 & Conventional heating & 240 & 68 & 0.35 & $\begin{array}{c}38 \\
\text { syn:anti }=16: 84\end{array}$ \\
\hline 6 & Conventional heating & 240 & 68 & 0.70 & $\begin{array}{c}74 \\
\text { syn:anti }=22: 78\end{array}$ \\
\hline 7 & Conventional heating & 240 & 68 & 1.40 & $\begin{array}{c}97 \\
\text { syn:anti }=9: 191\end{array}$ \\
\hline 8 & Conventional heating & 120 & 113 & 0.70 & $\begin{array}{c}58 \\
\text { syn:anti }=14: 86\end{array}$ \\
\hline
\end{tabular}

${ }^{\mathrm{a}}$ Solvent is pyridine: 1 -octanol $=1: 20$; ${ }^{\mathrm{b}}$ Conversion determined by $1 \mathrm{H}-\mathrm{NMR}$ integrated value. 
When acetophenone was used as the starting compound, the yield of oxime decreased significantly when the amount of hydroxylamine hydrochloride was reduced under conventional heating conditions. This was in contrast to that observed for acetyl ferrocene under microwave irradiation, indicating that acetylferrocene is susceptible to the microwave effect even under mild conditions.

It is evident from both the tables that conventional heating of acetylferrocene and acetophenone gave higher yields at a higher concentration of hydroxylamine hydrochloride (Entry 2 and 6, Table 1 and Table 2). However, when the amount of hydroxylamine hydrochloride was halved, the yield under microwave irradiation was (Entry 1) slightly higher than that under conventional heating conditions (Entry 5), regardless of the unfavorable microwave irradiation conditions. The difference in yield of acetylferrocene oxime was $15 \%$ when a high concentration of hydroxylamine hydrochloride was used (Entry 2 and 6). The difference was only $2 \%$ when a low concentration of hydroxylamine hydrochloride was used (Entry 1 and 5). Therefore, there was a microwave effect with a difference of $15 \%-2 \%=13 \%$. On the other hand, with acetophenone, the difference in yield was 9\% (Entry 2 and 6) and 7\% (Entry 1 and 5) at higher and lower concentrations of hydroxylamine hydrochloride, respectively. Therefore, there was a microwave effect with a difference of $9 \%-7 \%=2 \%$.

Under mild conditions, that is, low concentration of hydroxylamine hydrochloride, acetylferrocene showed a more prominent microwave effect. This is because the ferrocene nucleus in the molecule effectively absorbs microwaves [11] [12]. In addition, the yield of acetylferrocene oxime increased dramatically when the microwave output was set to $300 \mathrm{~W}$ (Entry 2 and 4), suggesting that acetylferrocene was easily affected by microwaves.

\subsection{Regioselectivity of Oximation}

Both syn and anti oximes were formed. The ratio of syn and anti forms is given in Table 1 and Table 2. There was no significant change in the ratio of syn and anti forms when the heating conditions or solvent polarity were changed.

\section{Conclusion}

In the oximation reaction of acetylferrocene, when the amount of hydroxylamine hydrochloride was halved, the yield decreased only slightly under microwave irradiation. However, the yield decreased by $20 \%$ under conventional heating conditions. On the other hand, in the oximation reaction of acetophenone, the yield decreased significantly under both microwave irradiation and normal heating conditions. From these facts, it can be seen that the ferrocene nucleus affects the microwave irradiation effect under mild conditions that are susceptible to the effect of microwaves. The efficient absorption of microwaves by the ferrocene nucleus was considered to accelerate the reaction. This therefore allows the oximation of ferrocene derivatives could be carried out at milder conditions at lower temperatures. Such a system could be useful as environmen- 
tally friendly synthesis.

\section{Conflicts of Interest}

The authors declare no conflicts of interest regarding the publication of this paper.

\section{References}

[1] Colacino, E., Dayaker, G., Morere, A. and Friscic, T. (2019) Introducing Students to Mechanochemistry via Environmentally Friendly Organic Synthesis Using a Solvent-Free Mechanochemical Preparation of the Antidiabetic Drug Tolbutamide. Journal of Chemical Education, 96, 766-771.

https://doi.org/10.1021/acs.jchemed.8b00459

[2] Coppock, P., Park, S.H., Paredes, J., Pennington, R., Pursell, D.P., Rudd, G., Sloop, J.C. and Tsoi, M.Y. (2017) Enhancing Research Skills and Attitudes in Undergraduate Organic Chemistry with a Course-Embedded Undergraduate Research Experience (CURE) via Green Organic Synthesis. Journal of Laboratory Chemical Education, 5, 41-47. http://article.sapub.org/10.5923.j.jlce.20170503.01.html

[3] Ilia, G., Iliescu, S. and Popa, A. (2015) Polymer-Supported Phase Transfer Catalysts in Green Organic Syntheses. Current Green Chemistry, 2, 264-273. https://doi.org/10.2174/2213346102999150306112846

[4] Sowmiah, S., Cheng, C.I. and Chu, Y. (2012) Ionic Liquids for Green Organic Synthesis. Current Organic Synthesis, 9, 74-95. https://doi.org/10.2174/157017912798889116

[5] Leveque, J. and Cravotto, G. (2006) Microwaves, Power Ultrasound, and Ionic Liquids. A New Synergy in Green Organic Synthesis. Chimia, 60, 313-320. https://doi.org/10.2533/000942906777836255

[6] Giguere, R., Bray, T., Duncan, S.M. and Majetich, G. (1986) Application of Commercial Microwave Ovens to Organic Synthesis. Tetrahedron Letters, 27, 4945-4948. https://doi.org/10.1016/S0040-4039(00)85103-5

[7] Mancini, P.M.E., Kneeteman, M.N., Cainelli, M., Ormachea, C.M. and Domingo, L.R. (2017) Nitropyrroles, Diels-Alder Reactions Assisted by Microwave Irradiation and solvent Effect. An Experimental and Theoretical Study. Journal of Molecular Structure, 1147, 155-160. https://doi.org/10.1016/j.molstruc.2017.06.109

[8] Du, C., Zhang, J., Li, L., Wang, K. and Luo, G. (2019) A Modified Mixed-Acid Catalytic System for Beckmann Rearrangement of Cyclohexanone Oxime. AIChE Journal, 65, e16603 https://doi.org/10.1002/aic.16603

[9] Lee, H. and Kim, H. (2014) Novel Fluorescent Probe for the Selective Detection of Organophosphorous Nerve Agents through a Cascade Reaction from Oxime to Nitrile via Isoxazole. Tetrahedron, 70, 2966-2970.

https://doi.org/10.1016/j.tet.2014.03.026

[10] Hayashi, T., Okada, Y. and Hukuchi, T. (1994) The Oxidative Decomposition Reaction of Acylferrocenes under Acidic Conditions. Nippon Kagaku Kaishi, No. 4, 340-344. https://doi.org/10.1246/nikkashi.1994.340

[11] Gao, Z., Zhang, J., Zhang, S., Lan, D., Zhao, Z. and Kou, K. (2020) Strategies for Electromagnetic Wave Absorbers Derived from Zeolite Imidazole Framework (ZIF-67) with Ferrocene Containing Polymers. Polymer, 202, 122679. https://doi.org/10.1016/j.polymer.2020.122679

[12] Ge, C., Zou, J., Yan, M. and Bi, H. (2014) C-Dots Induced Microwave Absorption Enhancement of PANI/Ferrocene/C-Dots. Materials Letters, 137, 41-44.

https://doi.org/10.1016/j.matlet.2014.08.111. 